\title{
A NOVEL KITE-CROSS-DIAMOND SEARCH ALGORITHM FOR FAST BLOCK MATCHING MOTION ESTIMATION
}

\author{
Chi-Wai Lam, Lai-Man $\underline{\text { Po }}$ and Chun Ho Cheung \\ Department of Electronic Engineering, City University of Hong Kong, Hong Kong SAR \\ cwlam@ee.cityu.edu.hk, eelmpo@cityu.edu.hk, terence@ieee.org
}

\begin{abstract}
In order to fit the small cross-center-biased characteristic of the real world video sequences, an improved version of the wellknown cross-diamond search algorithm (CDS) is proposed in this paper. Unlike traditional search pattern, such as square, diamond or cross - all are in vertically and horizontally symmetric shape, the kite-cross-diamond search (KCDS) algorithm adopts a novel asymmetric kite-shaped search patterns in the search step to keep similar distortion or even better in lowmotion sequence while the speed of the motion estimation for stationary or quasi-stationary blocks is further boosted. Experimental results show that this KCDS algorithm could achieve 58\% searching points reduction as compared with Diamond Search (DS) and $41 \%$ as compared with Cross Diamond Search (CDS) whereas similar prediction accuracy is preserved. Simulations show that KCDS is particularly faster and more accurate in some kinds of sequences. This algorithm is especially suitable for videoconferencing applications.
\end{abstract}

Keywords-Motion estimation, kite-cross-diamond search, cross-center biased characteristic.

\section{INTRODUCTION}

Motion estimation (ME) is a process to estimate the pels or pixels of the current frame from reference frame(s). Block matching algorithm (BMA), which is a temporal redundancy removal technique between 2 or more successive frames, is an integral part for most of the motion-compensated video coding standards. Frames are being divided into regular sized blocks, or so-called macroblocks (MB). Block-matching method is to seek for the best-matched block from the previous frame, usually the first single frame, within a fixed-sized of search window $(w)$. Based on a block distortion measure (BDM) or other matching criteria, the displacement of the best-matched block will be described as the motion vector (MV) to the block in the current frame. The best match is usually evaluated by a cost function based on a BDM such as Mean Square Error (MSE), Mean Absolute Error (MAE) or Sum of Absolute Differences (SAD). Full search (FS) method, which performs searching all the candidate blocks within the search window exhaustively, introduces high intensive computation. Over the last two decades, many fast motion estimation algorithms has been proposed to give a faster estimation with similar block distortion compared to FS. Some well known fast BMA are the three-step search (3SS) [1], the new three-step search (N3SS) [2], the four-step search (4SS) [3], the diamond search (DS) [4], the cross-diamond search (CDS) [5] and small cross-diamond search [6]. As the characteristic of center-biased motion vector distribution (MVD) which inspired many fast BMA in last decade, more than $80 \%$ of the blocks can be regarded as stationary or quasi-stationary blocks, i.e. most of the motion vectors are enclosed in the central $5 \times 5$ (blocks) area. This center-based characteristic can be even found in the fast-motion sequences. To exploits this phenomenon, N3SS added 8 center-neighboring blocks and introduced a halfway-stop technique to achieve crucial speedup for stationary and quasi-stationary blocks. 4SS also exploits the center-biased properties of motion vectors distribution by using halfway-stop techniques and smaller square search pattern compared to 3SS. DS was proposed with two novel ideas: a diamond shape searching pattern and unrestricted searching steps. DS is a highly center biased by using a compact diamond search pattern, and the unrestricted searching steps is used for reducing the chances of being trapped by local optima. In this paper, to fit cross-center -biased MVD property of the most real world sequences, a novel fast BMA called kite-cross-diamond search algorithm (KCDS) is proposed. It uses a small cross-shaped search patterns in the first step and results in higher speed for the motion estimation of stationary block. Similar starting pattern can be found in [7]. Then it uses a kite-shape pattern in second step to improve the accuracy in searching for quasi-stationary blocks. Experimental simulations show that it can achieve fewer search points over other BMAs, such as DS and CDS, and can obtain the similar distortion performance. The result also shows that it is favorable in videoconferencing sequences. This paper is organized as follows. The second section introduces the Cross -Center-Biased MVD property. The third section presents the details of the kitecross-diamond search algorithm. The fourth section describes the experimental result and some performance evaluations. Some concluding remarks are given in the last section.

\section{CROSS CENTER-BIASED MVP DISTRIBUTION}

\begin{tabular}{l|l}
\hline Frame Format (Numbers of frames) & \multicolumn{1}{c}{ Sequences } \\
\hline \hline CIF (352×288, 80 frames) & Miss America, Sales, Claire \\
\hline SIF (352×240, 80 frames) & Tennis, Garden, Football \\
\hline
\end{tabular}

TABLE I: Video Sequences used for Analysis

There is a well-known property of image sequences - The block motion field of a real world image sequence is usually gentle, smooth, and varies slowly. To demonstrate the property of the global minimum motion vector distribution, by applying FS with spiral block-matching style and MAD as the BDM on the six well-known real-world image sequences, which is listed in Table 1 , the average motion vector probabilities (MVP) distributions is tabulated in Table 2. The CIF sequences can be regarded as low motion (video conference) video, including "Miss America”, 
Probablities (\%) at corresponding checking-point within the search window

\begin{tabular}{|c|c|c|c|c|c|c|c|c|c|c|c|c|c|c|c|}
\hline $\mathrm{r}_{\mathrm{r} \text { Ver }}^{\mathrm{r} \text { HHor }}$ & -7 & -6 & -5 & -4 & -3 & -2 & -1 & 0 & 1 & 2 & 3 & 4 & 5 & 6 & 7 \\
\hline 7 & 62 & 2862 & 0.04252 & 02662 & 0.04 & .0543 & 0.03968 & 37227 & 0.03588 & 0.0441 & 02432 & 01885 & 0.03295 & \begin{tabular}{|l|}
0.01768 \\
\end{tabular} & 0.048 \\
\hline 6 & 0.013 & 327 & 0.00622 & 1483 & 0122 & 02285 & 0.04115 & 1553 & 0.05008 & 17 & 1527 & 0.00968 & 0.01053 & 0.0201 & 13 \\
\hline 5 & 0.006 & 0652 & 0.00517 & 0.00358 & 0.0 & 0.0239 & 0.0161 & 0.11638 & 0.05063 & 88 & 17 & $\overline{0.0}$ & 0.00412 & 0.0039 & $\overline{06}$ \\
\hline 4 & .03 & 345 & 0.0181 & 0.0342 & 0.6 & 23 & 0.11227 & 73 & 0.18613 & 83 & 342 & 0.02683 & 353 & .03798 & 0.019 \\
\hline 3 & 0.05 & 02778 & 0.04967 & 33 & 0.09733 & 25547 & 0.34103 & 27 & 0.35733 & 93 & 618 & .02578 & 895 & 0.02012 & 0.047 \\
\hline 2 & 0.015 & 02578 & 0.00643 & 02452 & 0.06072 & 0.25075 & 0.69938 & 24 & 0.41003 & 5 & 422 & 0.02737 & 748 & \begin{tabular}{|l|}
0.01957 \\
\end{tabular} & 0.023 \\
\hline 1 & 0.028 & .01832 & 0.02442 & 0.05295 & 0.1287 & 0.41457 & 0.738 & 4.216 & 0.623 & 0.2538 & 0.08102 & 0.04705 & 0.03682 & 0.0283 & 0.04 \\
\hline 0 & 0.161 & 0.36468 & 0.09355 & 0.22695 & 0.39983 & 0.814 & 4.378 & 45.49 & 3.44 & 0.516 & 0.30798 & 0.22548 & 0.11332 & \begin{tabular}{|l|}
0.25085 \\
\end{tabular} & 0.131 \\
\hline-1 & 0.028 & 0.00758 & 0.02525 & 0.04832 & 0.11068 & 0.39058 & 0.757 & 11.49 & 0.664 & 0.34932 & 0.10743 & 0.05103 & 0.03093 & 0.01305 & 0.029 \\
\hline-2 & 0.015 & 0.03313 & 0.01212 & 0.04733 & 0.07207 & 0.18257 & 0.38333 & 3.776 & 0.52453 & 0.24832 & 0.05852 & 0.03872 & 0.01843 & 0.02008 & 0.016 \\
\hline-3 & 0.045 & 0.02115 & 0.04695 & 0.0324 & 0.09555 & 0.25347 & 0.43328 & 2.95938 & 0.23842 & 0.23212 & 0.05493 & 0.03313 & 0.03808 & 0.02758 & 0.048 \\
\hline-4 & 0.015 & 0.03935 & 0.0118 & 0.02958 & 0.05093 & 0.07218 & 0.45172 & 0.67857 & 0.1452 & 0.10112 & 0.05567 & 0.03378 & 0.0181 & \begin{tabular}{|l|}
0.04398 \\
\end{tabular} & 0.013 \\
\hline-5 & 0.008 & 0.00547 & 0.00768 & 0.0098 & 0.01338 & 0.02935 & 0.64438 & 0.57385 & 0.05008 & 0.04937 & 0.01917 & 0.01935 & 0.01273 & 0.00812 & 0.01 \\
\hline-6 & 0.024 & 0.02063 & 0.01453 & 0.0159 & 0.01537 & 0.022 & 0.17688 & 0.29398 & 0.03218 & 0.03693 & 0.02747 & 0.02462 & 0.01525 & \begin{tabular}{|l|}
0.02598 \\
\end{tabular} & 0.011 \\
\hline-7 & 0.06 & 0.01463 & 0.036 & 0.01557 & 0.04535 & 0.04715 & 0.0524 & 0.35742 & 0.06335 & 0.06788 & 0.06135 & 0.06377 & 0.05252 & \begin{tabular}{|l|}
0.02537 \\
\end{tabular} & 0.068 \\
\hline
\end{tabular}

TABLE II: Average distribution measured at distance $r$ using 6 CIF/SIF sequences for $|w|=7$

"Salesman", and "Claire". These sequences is relatively gentle, smooth, and with low-motion content. Whereas another three SIF video sequences "Football", "Garden" and "Tennis" are relatively with high motion content. Zooming, fast movement object, and panning can be found in these 3 sequences. From observing the motion vector probabilities distributions on different sequences, we found that most real-world sequences possess the center-biased MVD characteristic (over 80\% MVP of the blocks having motion vectors within central $5 \times 5$ grid or radius- $r= \pm 2$ ), instead of an uniform distribution. The result also shows that the cross-center $M V$ distribution is more dominant within this radius. For instance, in Fig.1, $71.76 \%$ of the motion vectors are found located in the central $2 \times 2$ area, i.e., $\mathrm{A}+\mathrm{B}+\mathrm{D}$ or $r= \pm 1$. And there is about $68.98 \%$ of motion vector are located in $\mathrm{A}+\mathrm{B}$ or the cross-center region. Moreover, to look at $5 \times 5$ area $(\mathrm{A}+\mathrm{B}+\mathrm{C}+\mathrm{D}+\mathrm{E})$, total MVP is $81.75 \%$ and the cross-center probabilities within this area $(\mathrm{A}+\mathrm{B}+\mathrm{C})$ has accounted for $74.71 \%$. Furthermore, the probabilities sum of the 4 points in $\mathrm{E}$ (position within the cross) is higher than $\mathrm{D}$ (diagonal position). By observing the above analysis, we conclude that the cross-center distribution dominates the corresponding square region. Inspired from such a highly cross-center based distribution, the searching pattern of BMA in first few steps can be matched the crosscenter shape to save the searching point for stationary and quasistationary while maintain a similar distortion error. Another observation from the MVD is the effect of gravity - the vertical MVP down below the center point is holding a significant probability. e.g. the probability of $r_{-}$Ver $=-3, r_{-} H o r=0$ is almost 3\% whereas the opposite position in upper $\left(r_{-}\right.$Ver $=3$, $r_{\_}$Hor $=0$ ) is only $\sim 0.8 \%$.

\section{KITE-CROSS-DIAMOND SEARCH (KCDS) ALGORITHM}

In this section we first to describe the search patterns used in the algorithm, and later the search path strategy will be explained.

\section{A. Search Patterns}

The search-point configuration used in the KCDS are divided in 3 different shapes: cross-shaped pattern, diamond-shaped pattern and kite-shaped pattern. Fig.2 (a) show the small cross-shaped pattern (SCSP) and the large cross-shaped pattern (LCSP). The same search pattern from DS: small diamond-shaped pattern
(SDSP) and large diamond-shaped pattern (LDSP) are shown in Fig.2 (b).

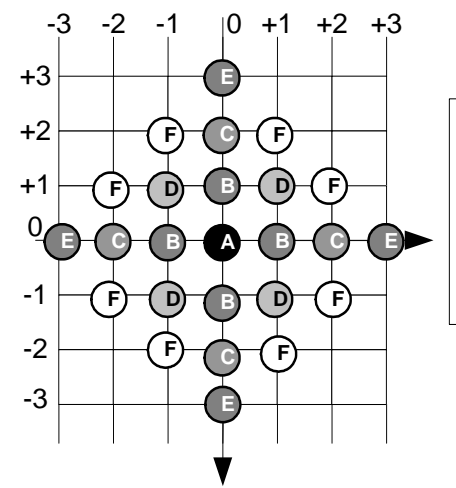
A: $45.49 \%$
B: Total: $23.52 \%$
C: Total :5.73\%
D: Total: $2.78 \%$
E: Total: $4.44 \%$
F: Total: $3.43 \%$

Fig. 1. The MVD within $r=4$ diamond shape area



( a )

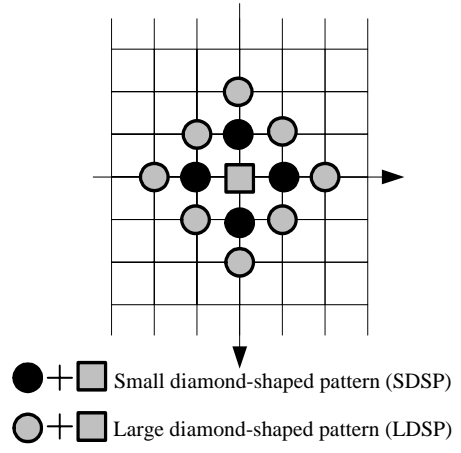

(b)
Fig. 2 Search Patterns used in the kite-cross-diamond search Unlike traditional search pattern, such as square, diamond, cross - all are vertically and horizontally symmetry, in the kiteshaped pattern (KSP), only the diagonal that connects the ends of the kite is the line of symmetry. Fig 3 (a) shows the vertical-kite by described as up-kite in which the dart (the most outer vertex) is pointing up. Fig 3 (b) is called left-kite. For another 2 KSP down-kite and right-kite are shown in Fig 3 (c) and (d). 


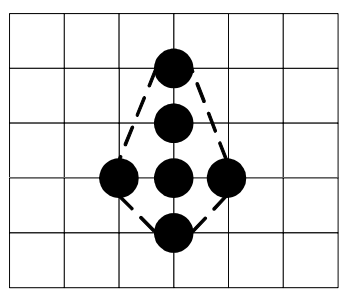

( a )

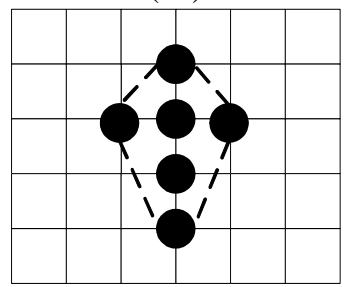

(c)

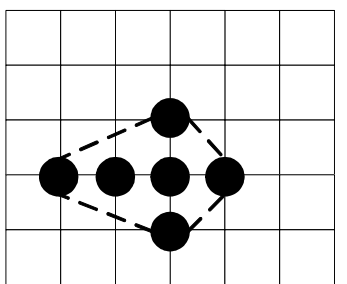

( b )

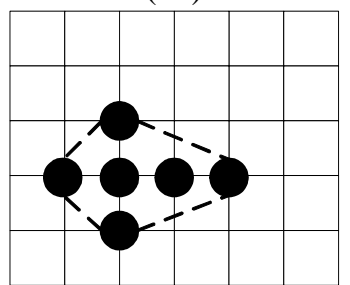

(d)
Fig. 3. Kite Search Patterns: With vertical symmetry (a) up-kite. and (c) down-kite; with horizontal symmetry: (b) left-kite and (d) right-kite.

\section{B. The KCDS algorihtm}

From the simulation result on those six well-known sequences, we found that nearly $70 \%$ blocks that can be regarded as stationary $(r=0)$ or quasi-stationary blocks $(r=1)$. For the sake of this highly small cross-center-biased property in most real world sequences, we take the small cross-shaped patterns as first step to the KCDS. The main difference between KCDS and cross diamond search (CDS) is that the first step of KCDS is a small cross-shaped pattern, which is saving the number of search point for stationary. And the KSP is employed in second step to improve the accuracy performance in quasi-stationary blocks by taking one more significant point (point E in Fig. 1) in the search pattern. The details and the analysis of the algorithm are given below:

Step 1 (Starting - Small Cross-Shape Pattern SCSP): A minimum BDM is found from the 5 search points of the SCSP (Small Cross-Shaped Pattern) [Fig.2 (a)] located at the center of search window. If the minimum BDM point occurs at the center of the SCSP $(0,0)$, the search stops (First Step Stop); otherwise, go to Step 2.

- Step 2 (KSP): With the vertex (minimum BDM point) in the first SCSP as the center, a particular KSP is formed based on the motion direction in previous step. For example, if the minimum BDM is located in upper vertex in first step, the new KSP will be an up-kite shape described as Fig 3 (a). Thus, there are 4 cases of newly formed KSP in this step: up-kite, down-kite, right-kite and left-kite, depends on the MV motion in step 1. If the minimum BDM point occurs at the center of this KSP, the search stops (Second Step Stop); otherwise go to Step 3.

- Step 3 (Diamond Searching): A new Large-Diamond-Shaped Pattern LDSP is formed by repositioning the minimum BDM found in previous step as the center of the LDSP. If the new minimum BDM point is at the center of the newly formed LDSP, then go to Step 4 for converging the final solution; otherwise, this step is repeated.

- Step 4 (Ending - Converging step): With the minimum BDM point in the previous step as the center, a SDSP (Small Diamond-
Shaped Pattern) is formed. Identify the new minimum BDM point from the SDSP, which is the final solution for the motion vector.
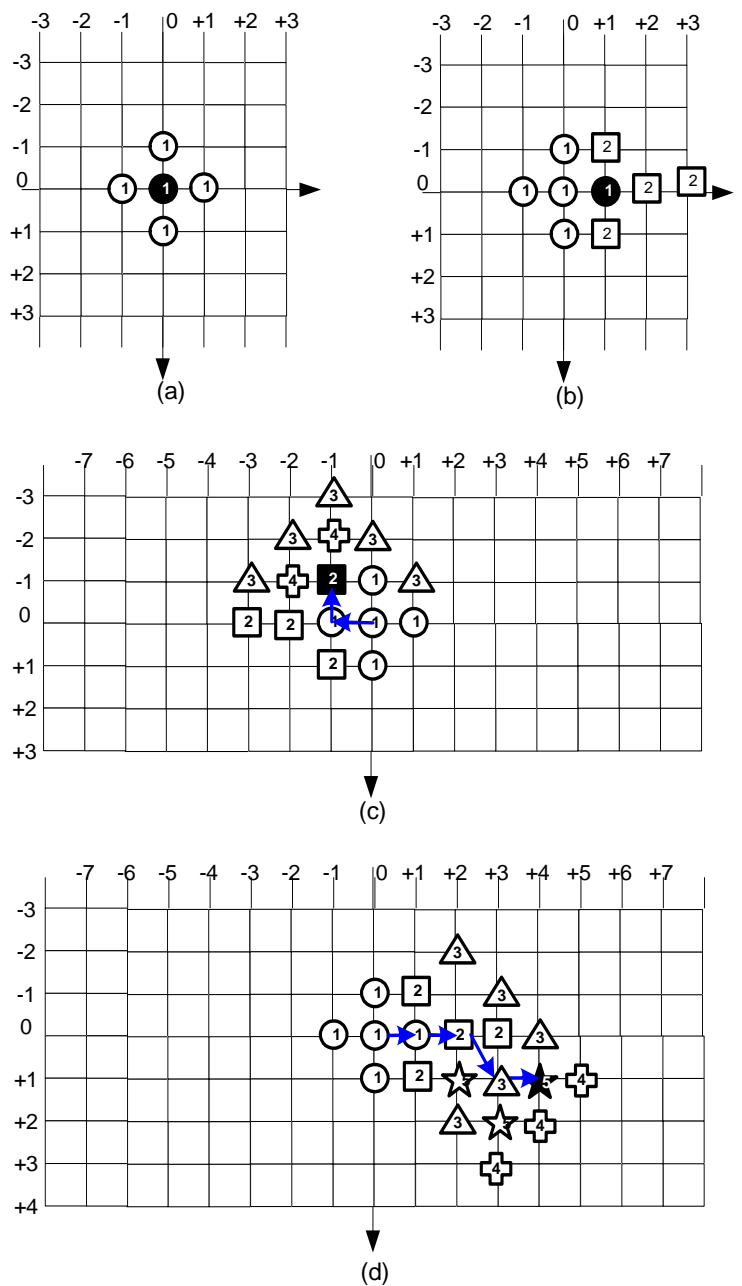

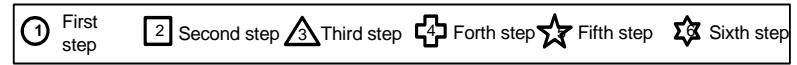

Fig. 4.Examples of the KCDS: (a) first-step-stop with $M V(0,0)$.

(b) Second-step-stop with $M V(1,0)$. (c) and (d)an unrestricted search path for $M V(-1,-1)$ and $(4,1)$ respectively.

\section{Analysis of KCDS algorithm}

KCDS is regarded as an improved version of CDS because both of them also focus on advancing the speed and quality performance of videoconferencing sequences. To compare the CDS and the DS, the main improvement of this algorithm is the speed performance (the number of searching point). KCDS reduces the number of search points significantly if there is stationary or quasi-stationary blocks. The configuration of the searching patterns is to fit the small cross-center-biased MV distribution characteristics. Thus, it provides more chance to save up the searching points for motion vectors. And the kite shape pattern in step 2 improve the quality by searching one more point on the $r= \pm 3$ (vertical or horizontal vertex of a cross). In Fig 4, it shows 4 typical examples of KCDS and each candidate point is marked with the corresponding step number. Fig.4 (a) and (b) show the two halfway-stop examples. The KCDS only takes 5 (first step stop) and 9 (second step stop), 
whereas the CDS took 9 and 11 search points, and the DS took 13 search points for searching the same block respectively. Another two search paths for $r>1$ are shown in Fig 4 (c) and (d). After step 3, the subsequent steps will be exactly the same as diamond search. To provide faster and more accurate solution, this algorithm emphasis on the speed of video conferencing application, which is gentle and small motion.

\section{EXPERIMENTAL RESULTS}

In our simulations, the mean absolute error (MAE) used as the BDM. The block size is at $16 \times 16$, and the maximum displacement in the search areas is \pm 7 pixels in both the horizontal and the vertical directions. The simulation is performed with a total of six sequences with different degrees and types of motion content as listed in Table I. We compared the KCDS against DS, CDS and SCDS using the following test criteria: 1) Average searching point (ASP) - the average number of search point used to find the motion vector; and 2) Average MAE per pixel- This shows the magnitude of distortion per pixel. Table 3 and 4 summarize the experimental results of each search strategy over the test criteria using the 6 tested sequences. And the speed/MAD improvement in percentage of the KCDS over DS and CDS are tabulated in Table V. By observing the result obtained in Table III, The KCDS takes the smallest average number of search points per block - KCDS $<$ SCDS $<$ CDS $<$ DS $<$ N3SS $<$ 3SS $<$ FS (i.e. the fastest) among other fast BMA for all of the six test sequences. To compare with DS, among the video conferencing sequence, such as "Miss America”, "Sales", and "Claire", the proposed KCDS obtains at least $41 \%$ of speed improvement, even in vigorous motion content like "Football", the speed up ratio can be achieved up to $43 \%$ and the worst is $22 \%$ on Tennis sequence. The trade off of the block distortion for faster speed is tabulate in Table IV which compare the difference of average MAE per pixel from FS. The result shows that the KCDS gives nearly the same MAE performance as compared to CDS in most sequences. In some sequences KCDS even perform better, such as "Miss America” and "Sales), In which it results around $1.1 \%$ up to $2.8 \%$ of the quality improvement compared to DS. For high motion content, the KCDS introduce slight quality degradation as compared to DS and CDS (maximum $~ 5 \%$ of the degradation in "Tennis" with the trade off of at least $17 \%$ speed improvement). Therefore, in video conferencing sequences, KCDS performs excellent, in which this is the fastest among all BMA and more accurate compared to DS and CDS in some sequences. For high motion sequence, it still maintains a satisfying tradeoff between error distortion and speedup ratio.

\section{CONCLUSION}

By observing the cross-center biased motion vector distribution characteristics of the real world video sequences, we proposed a kite-cross-diamond search algorithm (KCDS), which emphasis a novel idea of kite shape pattern. Simulation results showed that KCDS is the fastest algorithm among the testing BMA while providing similar prediction accuracy. It is especially suitable for videoconferencing sequences.

\section{ACKNOWLEDGMENT}

The work described in this paper was substantially supported by a grant from City University of Hong Kong, Hong Kong SAR, China. [Project No.7001385]

\begin{tabular}{l|l|l|l|l|l|l|l|r}
\multicolumn{10}{c}{ Average searching point ASP } \\
\hline \hline & FS & $3 S S$ & 4 SS & N3SS & DS & CDS & SCDS & KCDS \\
\hline Tennis & 202.1 & 23.20 & 18.65 & 20.67 & 16.25 & 15.38 & 13.9 & $\mathbf{1 2 . 6 7}$ \\
\hline Garden & 202.1 & 23.24 & 18.80 & 21.38 & 16.84 & 15.09 & 14.87 & $\mathbf{1 2 . 8 9}$ \\
\hline Football & 202.1 & 23.06 & 16.69 & 17.65 & 13.67 & 10.96 & 8.24 & $\mathbf{7 . 7 3}$ \\
\hline MissA & 202.1 & 23.46 & 18.319 & 19.99 & 16.36 & 11.75 & 10.75 & $\mathbf{9 . 5 4}$ \\
\hline Claire & 202.1 & 23.22 & 15.924 & 16.19 & 12.4 & 8.92 & 5.38 & $\mathbf{5 . 2 3}$ \\
\hline Sales & 202.1 & 23.21 & 16.206 & 16.94 & 13.02 & 9.5 & 6.99 & $\mathbf{6 . 4 3}$ \\
\hline
\end{tabular}

TABLE III: The Average number of searching points of FS,3SS, 4SS, N3SS, DS, CDS, SCDS, and KCDS over the six sequences

Difference of average MAE per pixel from FS

\begin{tabular}{l|l|l|l|l|l|l|r}
\hline \hline & 3SS & 4 SS & N3SS & DS & CDS & SCDS & \multicolumn{1}{|c}{ KCDS } \\
\hline Tennis & 1.0374 & 0.4383 & 0.488 & 0.2415 & 0.2935 & 0.3584 & $\mathbf{0 . 5 3 8 4}$ \\
\hline Garden & 0.9845 & 0.6502 & 0.1568 & 0.2337 & 0.1906 & 0.2056 & $\mathbf{0 . 2 2 4 1}$ \\
\hline Football & 0.2436 & 0.1683 & 0.1034 & 0.1452 & 0.1709 & 0.19 & $\mathbf{0 . 2 3 3}$ \\
\hline MissA & 0.1169 & 0.1165 & 0.0253 & 0.1021 & 0.0352 & 0.0371 & $\mathbf{0 . 0 3 5 1}$ \\
\hline Claire & 0.0038 & 0.0035 & 0.001 & 0.0014 & 0.0029 & 0.0033 & $\mathbf{0 . 0 0 3 2}$ \\
\hline Sales & 0.0521 & 0.044 & 0.0081 & 0.0423 & 0.0094 & 0.01 & $\mathbf{0 . 0 0 9 4}$ \\
\hline
\end{tabular}

TABLE IV: Differences of average MAE per pixel from FS of FS,3SS, 4SS, N3SS, DS, CDS, SCDS, and KCDS over the six sequences

\begin{tabular}{l|r|r|r|r}
\multicolumn{2}{c}{} & \multicolumn{2}{c|}{ KCDS over DS } & \multicolumn{2}{c}{ KCDS over CDS } \\
\hline \hline & SIR (\%) & MAE & \multicolumn{1}{c}{ SIR } & MAE \\
\hline Tennis & -22.0086 & 5.242163 & -17.602 & 4.283191 \\
\hline Garden & -23.4594 & -0.11086 & -14.6113 & 0.383608 \\
\hline Football & -43.4483 & 1.333434 & -29.494 & 0.938378 \\
\hline MissA & -41.6906 & -2.81099 & -18.8443 & -0.00432 \\
\hline Claire & -57.8271 & 0.163871 & -41.3384 & 0.019251 \\
\hline Sales & -50.5949 & -1.13531 & -32.263 & -0.00348 \\
\hline
\end{tabular}

TABLE V: Average Speed Improvement ratio and average MAE changed percentage of the KCDS over DS and CDS

\section{REFERENCES}

[1] T. Koga, K. Iinuma, A. hirano, Y. Iijima, and T. Ishiguro, "Motion compensated interframe coding for video conferencing”, in Proc. Nat. Telecommun. Conf., New Orleans, L.A., Nov.-Dec. 1981, pp. G5.3.1-G5.3.5.

[2] R. Li, B. Zeng, and M. L. Liou, “A new three-step search algorithm for block motion estimation”, IEEE Trans. Circuits Syst. Video Technol., vol. 4, pp. 438-443, Aug 1994.

[3] L. M. Po and W. C. Ma, “A novel four-step search algorithm for fast block motion estimation”, IEEE Trans. Circuits Syst. Video Technol., vol. 6, pp. 313-317, Jun 1996.

[4] J. Y. Tham, S. Ranganath, M. Ranganath, and A. A. Kassim, “A novel unrestricted center-biased diamond search algorithm for block motion estimation”, IEEE Trans, Circuits Syst. Video Technol., vol. 8, no. 4, pp. 369-377, Aug 1998.

[5] C. H. Cheung, and L. M. Po, “A Novel Cross-Diamond Search Algorithm for Fast Block Motion Estimation”, IEEE Trans, Circuits Syst. Video Technol., vol. 12, no. 12, Dec 2002.

[6] C. H. Cheung and L. M. Po, "A novel small-cross-diamond search algorithm for fast video coding and videoconferencing applications”, in Proc. IEEE ICIP, Sept. 2002.

[7] Yao Nie; Kai-Kuang Ma, "Adaptive rood pattern search for fast block-matching motion estimation”, IEEE trans. Image Processing, vol. 11, pp.1442-1449, Dec 2002. 